\title{
In Vivo Development of Outer Retinal Synapses in the Absence of Glial Contact
}

\author{
Philip R. Williams, ${ }^{1,3}$ Sachihiro C. Suzuki, ${ }^{1}$ Takeshi Yoshimatsu, ${ }^{1}$ Owen T. Lawrence, ${ }^{2}$ Steven J. Waldron, ${ }^{1}$ \\ Michael J. Parsons, ${ }^{5}$ Michael L. Nonet, ${ }^{4}$ and Rachel 0. L. Wong ${ }^{1}$ \\ Departments of ${ }^{1}$ Biological Structure and ${ }^{2}$ Biochemistry, University of Washington, Seattle, Washington 98195, ${ }^{3}$ Program in Neurosciences and \\ ${ }^{4}$ Department of Anatomy and Neurobiology, Washington University, St. Louis, Missouri 63110, and ${ }^{5}$ Department of Surgery, Johns Hopkins University, \\ Baltimore, Maryland 21205
}

Astroglia secrete factors that promote synapse formation and maintenance. In culture, glial contact has also been shown to facilitate synaptogenesis. Here, we examined whether glial contact is important for establishing circuits in vivo by simultaneously monitoring differentiation of glial cells and local synaptogenesis over time. Photoreceptor circuits of the vertebrate retina are particularly suitable for this study because of the relatively simple, laminar organization of their connectivity with their target neurons, horizontal cells and bipolar cells. Also, individual photoreceptor terminals are ensheathed within the outer plexiform layer (OPL) by the processes of one type of glia, Müller glia cells (MGs). We conducted in vivo time-lapse multiphoton imaging of the rapidly developing and relatively transparent zebrafish retina to ascertain the time course of MG development relative to OPL synaptogenesis. The emergence of synaptic triads, indicative of functional photoreceptor circuits, and structural association with glial processes were also examined across ages by electron microscopy. We first show that MG processes form territories that tile within the inner and outer synaptic layers. We then demonstrate that cone photoreceptor synapses are assembled before the elaboration of MG processes in the OPL. Using a targeted cell ablation approach, we also determined whether the maintenance of photoreceptor synapses is perturbed when local MGs are absent. We found that removal of MGs had no appreciable effect on the stability of newly formed cone synapses. Thus, in contrast to other CNS circuits, contact from glia is not necessary for the formation or immediate stabilization of outer retinal synapses.

\section{Introduction}

Functional synapses involve not only presynaptic and postsynaptic neurons but also surrounding glia. Not only are glia involved in synapse maintenance (Ransom et al., 2003), but recent studies also demonstrate that glia promote synapse development and plasticity (Stevens, 2008). For example, ablation of perisynaptic Schwann cells arrests neuromuscular junction maturation and often results in the retraction of the nerve terminal (Reddy et al., 2003). Also, glial differentiation coincides with increased synaptogenesis in the superior colliculus (Ullian et al., 2001). But, in some regions of the nervous system, differentiation of glial cells occurs only after functional circuits emerge. Notably, cortical astrocytes differentiate (Müller, 1990; Rochefort et al., 2005) well after synaptogenesis and the establishment of functional connections between thalamic neurons and the cortical subplate (Friauf et al., 1990). However, subplate neurons eventually die, raising the possibility that perhaps glia are important for the forma-

Received June 30, 2010; accepted July 15, 2010.

This work was supported by National Institutes of Health Grants EY14358 and EY01730 and the Uehara Memorial Foundation (S.C.S., T.Y.). We thank Steve Leach for the $T g\left(\right.$ ptf1a:Gal4VP16) ${ }^{\text {ih16 }}$ transgenic zebrafish, Pamela Raymond for the $\mathrm{Tg}$ ( gfap:GFP) ${ }^{\text {mizoor }}$ transgenic zebrafish, Shoji Kawamura for the SWS1:GFP plasmid, Teresa Nicolson for the antibody to RIBEYEb, Ed Parker for electron microscopy, Jing Huang for cryosectioning, and Felice Dunn for critical reading of this manuscript.

Correspondence should be addressed to Rachel 0. L. Wong, Department of Biological Structure, University of Washington, Box 357420, Seattle, WA 98195. E-mail:wongr2@u.washington.edu.

DOI:10.1523/JNEUROSCI.3391-10.2010

Copyright $\odot 2010$ the authors $\quad 0270-6474 / 10 / 3011951-11 \$ 15.00 / 0$ tion and maintenance of persisting, rather than transient, connections.

The influence of glia could involve both secreted factors and contact-mediated signaling. Although it is now well established that glia secrete numerous synapse-promoting factors (Mauch et al., 2001; Ullian et al., 2001, 2004; Beattie et al., 2002; Christopherson et al., 2005; Feng and Ko, 2008), a role for direct glial-neuronal contact has only recently been assessed (Liu et al., 1996; Hama et al., 2004; Nishida and Okabe, 2007). In particular, in hippocampal cultures, coordinated motility of astrocytic processes and dendritic filopodia facilitates glial-neuronal contact (Haber et al., 2006), and increases the likelihood of spine formation (Nishida and Okabe, 2007).

Here, we determined the developmental relationship between glial differentiation and synapse assembly in a retinal network that is maintained for adult function using live-cell imaging approaches. In addition, we specifically addressed whether glialneuronal contact is necessary for establishing outer retinal connections during development in vivo. We focused on retinal Müller glial cells (MGs), which have processes that associate with synapses in the mature retinal synaptic layers, the inner and outer plexiform layers (IPL and OPL). In particular, we investigated the relationship between process outgrowth of MGs and photoreceptor synaptogenesis, largely because these synapses in the OPL are stereotypic and readily visualized. To do so, it was necessary to follow the early elaboration of MG processes into the future synaptic layers. Early MG differentiation is not detectable by marker proteins available to date. But recently, the availability of a trans- 
genic zebrafish line (Bernardos and Raymond, 2006) in which MGs express green fluorescent protein (GFP) during their early stages of differentiation provided a means for us to perform in vivo time-lapse multiphoton microscopy to directly correlate MG differentiation with synaptogenesis in the retina. Because retinal circuits in zebrafish develop relatively quickly (within days), it was also possible to determine whether local removal of MGs affected photoreceptor synapse arrangements over time. This was achieved by performing targeted MG ablation in vivo using the multiphoton laser. Our observations suggest that unlike other parts of the nervous system, photoreceptor circuits essential for vision are established independent of glial cell contact.

\section{Materials and Methods}

Transgenic zebrafish. Zebrafish were maintained in accordance with University of Washington Institutional Animal Care and Use Committee guidelines. We used a combination of transgenic lines to visualize MGs, ON bipolar cells, and horizontal cells (HCs). In the transgenic line $T g$ ( gfap:GFP) ${ }^{\text {mizool }}$ (referred to as gfap:GFP), all MGs express GFP (Bernardos and Raymond, 2006). In the transgenic line $\operatorname{Tg}($ nyx:Gal4VP16; $U A S: M Y F P)^{Q 16}$ (referred to as Q16), a majority of ON bipolar cells indirectly express YFP fused to the palmitoylation signal of gap43 (MYFP) for membrane targeting (Schroeter et al., 2006). In the transgenic line $T g$ ( $p t f 1 a$ :Gal4-VP16) ${ }^{\text {jh16 }}$ (referred to as ptf1a:G4VP16), most if not all HCs express the transcription factor Gal4VP16 (Parsons et al., 2009).ptf1a:G4VP16 transgenic fish were crossed to $\mathrm{Tg}$ (pBleeding Heart $U A S: M Y F P$ ) transgenic zebrafish (referred to as UAS:MYFP) to label the vast majority of HCs with MYFP. In the UAS:MYFP transgenic line, the cardiac myosin promoter drives the expression of mCherry in the heart (Huang et al., 2003) for ease of screening. These zebrafish were created by coinjecting the pBleedingHeart UAS:MYFP plasmid with Tol2 mRNA to increase the efficiency of transgene integration (Kawakami, 2004). F0 founders were screened by expression of mCherry in the heart, and UAS: MYFP activity was confirmed by crossing F1 zebrafish with Gal4VP16expressing transgenic lines, where MYFP expression was found to be near complete. All transgenic lines were mated into the roy orbison mutant background (Ren et al., 2002) to prevent iridophore formation, thus allowing for in vivo imaging of the retina.

Plasmid production and cloning. gfap:cerulean was generated using standard cloning techniques. The GFP encoding region was excised from gfap:GFP (Bernardos and Raymond, 2006) using NotI and KpnI restriction enzymes. A DNA fragment encoding cerulean with NotI and KpnI restriction sites was obtained by restriction digest of a ceruleancontaining vector (Livet et al., 2007), and ligated with the backbone of gfap:GFP generated above.

To isolate the essential promoter elements of thyroid hormone receptor $\beta 2$ $(\operatorname{tr} \beta 2)$, a $1.8 \mathrm{~kb}$ upstream and a $2.0 \mathrm{~kb}$ downstream genomic fragment flanking exon 1 of $\operatorname{tr} \beta 2$ were amplified from the zebrafish BAC clone, CH211-175H19, using the following primers: upstream fragment: 5' -GGGGACAACTTTGTATAGAAAAGTTGGACTCTGGTGTTGAGGGGCTTT-3' and 5'-GGGGACTGCTTTTTTGTACAAACTTGCACCGCATATCCGCCACTTACAC-3'; downstream fragment: 5' -AAAAATCGATAGGGCCACATAAGAAAGGTATTGC-3' and 5' AAAGGATCCACAGCTCATCCTTGTCCAGGTAAC-3'. The fragments were combined with the tdTomato reporter using the Gateway-based tol2kit (Kwan et al., 2007).

SWS1:tdTomato was also generated by modifying the original constructs (Takechi et al., 2003; Schroeter et al., 2006) using the Gatewaybased tol2kit (Kwan et al., 2007).

pBleeding Heart multicloning site (mcs) was made to facilitate the construction of transgenic zebrafish with no inherent fluorophore expression. Two kilobases of extraneous medaka tyrosinase genomic DNA between MscI and NgoMI restriction sites were removed from T2KSAG (Kawakami and Shima, 1999). We then inserted a 246 bp heart-specific promoter element, cmlc2 (Huang et al., 2003), driving mCherry between the left arm of Tol2 and the bidirectional SV40 polyadenylation signal present in the parent vector. An mcs polylinker was inserted in place of the GFP cassette to provide convenient restriction sites for cloning.
pBleeding Heart UAS:MYFP was then constructed by ligating the promoter and coding region of UAS:MFYP (Schroeter et al., 2006) into the mcs of pBleeding Heart mcs.

Transient expression of fluorescent cell labels. Mosaic expression of fluorophores in retinal cells was performed by injecting plasmid cDNA into one-cell stage zebrafish embryos as previously described (Schroeter et al., 2006). Briefly, a pulled-glass micropipette loaded with DNA was attached to a Picospritzer II (Parker) and anchored on a micromanipulator (Narishige) for small volume microinjections. Injection pressure was set to 10 Psi and durations ranged from 100 to $1000 \mathrm{~ms}$. Zebrafish embryos were arrayed in custom-made silicon molds and oriented with their cells upward to facilitate injections. gfap:GFP and $g f a p$ :Cerulean were used to visualize MGs (Bernardos and Raymond, 2006) and SWS1:tdTomato (Takechi et al., 2003) and $\operatorname{tr} \beta 2:$ tdTomato were used to visualize cone photoreceptors. Coinjection of Cx55.5:Gal4VP16 and UAS:MCFP was used to visualize HCs (Godinho et al., 2007). Tubulin:Gal4VP16 (Köster and Fraser, 2001) and UAS:MCerulean was coinjected to visualize neurons. DNA concentrations from 5 to $20 \mathrm{ng} / \mu$ l diluted in $1 \times$ Danieau's solution [ $58 \mathrm{~mm} \mathrm{NaCl}, 0.7 \mathrm{~mm} \mathrm{KCl}, 0.6 \mathrm{~mm} \mathrm{Ca}\left(\mathrm{NO}_{3}\right)_{2}, 0.4 \mathrm{~mm} \mathrm{MgSO}_{4}, 5$ mм HEPES, pH 7.6] with $0.1 \%$ phenol red to aid injection bolus visualization were used for the injections. At 10-12 h postfertilization (hpf), embryos were placed in $0.3 \times$ Danieau's solution with $0.2 \mathrm{~mm}$ propylthiouracil (PTU) to prevent melanophore pigmentation.

DiI labeling. gfap:GFP larvae [3-5 d postfertilization (dpf)] were anesthetized in $0.3 \times$ Danieau's solution with $0.02 \%$ tricaine and $0.2 \mathrm{~mm}$ PTU. They were then embedded in $0.5 \%$ low-melting-point agarose in the solution above. A small glass pipette ( $1 \mu \mathrm{m}$ tip size) was inserted into the eye and 1,1' -dioctadecyl-3,3,3',3' -tetramethylindocarbocyanine perchlorate (DiI; Invitrogen; $<0.6 \mathrm{pl}, 2 \mathrm{mg} / \mathrm{ml}$ stock dissolved in dimethyl sulfoxide) was injected using a pressure injector (Picospritzer II). DiI was allowed to diffuse for at least $1 \mathrm{~h}$ at $28.5^{\circ} \mathrm{C}$ in the anesthetized fish before imaging.

Multiphoton live-cell imaging. Embryos were prepared for in vivo imaging as previously described (Godinho et al., 2005). Briefly, transparent embryos were mounted in molten $40^{\circ} \mathrm{C}, 0.5 \%$ low-melting-point agarose (type VII, Sigma) with $0.02 \%$ tricaine anesthesia and $0.2 \mathrm{~mm}$ PTU in 60 $\mathrm{mm}$ organotypic culture dishes (Falcon). After the agarose set for $30 \mathrm{~min}$, samples were flooded with $0.3 \times$ Danieau's solution containing $0.02 \%$ tricaine and 0.2 mM PTU. Multiphoton image stacks were acquired on a custom-built two-photon microscope consisting of an FV300 scanhead (Olympus) and a Ti-Sapphire tunable infrared laser (Spectra-Physics). Laser intensity was measured as it entered the scanhead and ranged from 15 to $100 \mathrm{~mW}$ depending on the experiment. The Ti-Sapphire laser was tuned to $845-860 \mathrm{~nm}$ for imaging Cerulean/CFP and GFP, $880 \mathrm{~nm}$ for imaging GFP, $890 \mathrm{~nm}$ for imaging GFP and YFP, and $890-910 \mathrm{~nm}$ for imaging GFP and tdTomato. A 1.1 NA $60 \times$ water-immersion objective with a correction collar was used (Olympus). Zebrafish embryos were released from agarose and returned to a $28.5^{\circ} \mathrm{C}$ incubator between 6 or $24 \mathrm{~h}$ time points.

Photobleaching. Individual MGs were photobleached on the multiphoton microscope by scanning a small region of interest $\left(<0.5 \mu \mathrm{m}^{2}\right)$ over the cell soma at twice normal laser acquisition intensity for $30-45 \mathrm{~min}$ as needed. Photobleaching was verified and, if required, further scanning was allowed for increments of $10 \mathrm{~min}$ until fluorescence was appreciably diminished.

Müller glia ablations. MGs were ablated using the multiphoton laser as previously described for mouse retinal HCs (Huckfeldt et al., 2009). The laser wavelength was adjusted to $750 \mathrm{~nm}$ to prevent photobleaching, the digital zoom was increased to the maximum, and a small line scan across the apical process of the MG close to the soma was performed 10 times. If the process was not severed, the line scan was repeated until changes in the signal were observed. Severed processes were indicated by either an increase in fluorescence intensity, indicating damaged autofluorescent material, or loss of fluorescence, indicating that the GFP-containing process was no longer continuous within the scanned region. This resulted in the death of most MGs observed. In instances in which the MGs survived apical process ablation, apical process regrowth into the OPL was not seen. This procedure was repeated 6-8 times on adjacent MGs to create a zone free of MG processes in the OPL. 
Immunofluorescence and confocal microscopy. Transgenic zebrafish (78 hpf) were overdosed with $0.4 \%$ tricaine and fixed with $4 \%$ paraformaldehyde and 2\% sucrose in PBS for 20 min. Samples were washed three times for $5 \mathrm{~min}$ in PBS. For immunolabeling in frozen sections, the samples were then immersed in sucrose using a $5 \%, 10 \%$, and $15 \%$ series for 30 min each. Fish were then sunk overnight in 20\% sucrose in PBS. Samples were frozen in OTC and $20 \mu \mathrm{m}$ coronal sections were cut using a freezing microtome. Sections were washed three times with PBS and blocked for $1 \mathrm{~h}$ in blocking solution containing $0.3 \%$ Triton X-100 and $5 \%$ normal donkey serum in PBS. Primary antibodies against GluR2/3 (rabbit, 1:400, Millipore) and RIBEYEb [rabbit, 1:200 (Obholzer et al., 2008)] were diluted in blocking solution and incubated on slides overnight at room temperature. Following three washes with PBS, secondary antibody (goat-anti rabbit-conjugated Alexa Fluor 568, 1:1000, Invitrogen) diluted in blocking serum was applied to samples for $1 \mathrm{~h}$. Samples were washed three times and slides were coverslipped with VectaShield (Vector Laboratories). For immunostaining with anti-GFAP (zrf-1; ZIRC, Oregon) on whole animals, 1:200 dilution of the primary antibody was used and the secondary antibody was Alexa Fluor 568-conjugated goat anti-mouse $\operatorname{IgG1}(\gamma 1)$ (1:1000, Invitrogen).

Confocal image stacks were acquired on a FV1000 confocal microscope using a 1.35 NA $60 \times$ oil-immersion objective (Olympus). A solidstate laser $(440 \mathrm{~nm})$, multiline argon laser $(488 \mathrm{~nm})$ and a krypton laser $(568 \mathrm{~nm})$ were used for excitation.

Electron microscopy. Zebrafish embryos/larvae were anesthetized in $0.02 \%$ tricaine, in embryo medium, neutral $\mathrm{pH}$, killed on ice, and rapidly fixed in $4 \%$ glutaraldehyde $/ 2 \%$ paraformaldehyde in $0.1 \mathrm{M}$ sodium cacodylate buffer, $\mathrm{pH} 7.4$ for $3 \mathrm{~h}$ at $4^{\circ} \mathrm{C}$. The tissue was then washed in buffer, fixed in $1 \%$ osmium tetroxide in cacodylate buffer for $1 \mathrm{~h}$ and then en bloc stained with uranyl acetate (1\%). Thereafter, the tissue was dehydrated in a graded ethanol series, embedded in Araldite resin, thin sectioned, and poststained with $1 \%$ lead citrate.

To determine whether photoreceptors possessed ribbons at $77 \mathrm{hpf}$, we reconstructed photoreceptors in serial sections across the OPL, spanning a width of $\sim 75 \mu \mathrm{m}$ and a total depth of $0.5 \mu \mathrm{m}$. To ensure that the sections encompass a sufficient volume of the cone pedicle, we selected cone photoreceptors for which a minimum pedicle width of $2 \mu \mathrm{m}$ was found within the sections (cone photoreceptors at $77 \mathrm{hpf}$ possessed a maximum diameter between 2.5 and $3 \mu \mathrm{m}$ ).

Data analysis. Images were median filtered and levels were set manually using MetaMorph software (Medical Devices). Amira software (Visage Imaging) was used for three-dimensional (3D) reconstructions and to spatially align time-lapse series. For retinal layer measurements, 2D projections of retinal layers were created in Amira by first properly aligning the $3 \mathrm{D}$ stacks and creating oblique slices above and below the regions of interest. Measurements were performed from these 2D projections using MetaMorph software. Percentage coverage was measured by manually thresholding the image and dividing the number of pixels above threshold by the total number of pixels in the area. Photobleached and ablated areas were measured using the freeform region of interest tool. Centers of mass for MG arbors were calculated by assigning vector coordinates to each pixel in an image, and then measuring and averaging coordinates for all pixels within an arbor (Matlab).

Time-lapse imaging of $\mathrm{HC}$ dendritic tips was analyzed using Amira 3D reconstructions of the cells for each time point. Dendritic tips present at each time point were first marked using the label field tool to display dendritic tips as a single point. Then tips were determined to be present or absent in previous time points by comparing label fields and $3 \mathrm{D}$ reconstructions across time. Dendritic tips were scored as contacting or not contacting $\mathrm{MG}$ processes in these $3 \mathrm{D}$ reconstructions.

Photoreceptor invaginations were determined both by scrolling through the image stack plane by plane and by examining the 3D reconstruction. Photoreceptor-MG contacts were scored by scrolling through dual color image stacks and using $3 \mathrm{D}$ rotations in Amira. HC tips in the OPL were counted using the label field tool in Amira. Areas of the OPL devoid of MG processes in ptf1a:Gal4VP16; UAS:MYFP transgenic zebrafish were calculated by measuring the lateral distance lacking $M G$ processes in each image stack and multiplying the sum by the $z$-plane thickness. Only areas of the OPL large enough to contain six or more HC dendritic tips in the normal retina $\left(25 \mu \mathrm{m}^{2}\right)$ were analyzed. MannWhitney $U$ test and Fisher's exact test were used for calculations of statistical significance.

\section{Results}

Before assessing the developmental relationship between MGs and outer retinal synapses, we first characterized the morphology and distribution of MG processes in the zebrafish retina throughout the differentiation of these cells. Next, we asked how ingrowth of MG processes into the OPL relates to synaptogenesis and stability of newly formed synapses in this layer. Then, to directly assess the importance of MGs in regulating synapse development in the OPL, we locally ablated small numbers of MGs in vivo and performed time-lapse imaging studies to monitor the development of photoreceptor terminals and their postsynaptic targets in regions where MGs were absent.

\section{Processes of mature zebrafish MGs occupy exclusive territories within the synaptic layers}

Isolated MGs were visualized in vivo by transient expression of GFP just after they had completed arbor elaboration at $\sim 5 \mathrm{dpf}($ Fig. $1 \mathrm{~A}$ ). At this stage, $M G$ processes terminated and stratified at the outer and inner limiting membranes (OLM, ILM) as well as the synaptic layers of the retina (Fig. 1) (see also Bernardos and Raymond, 2006), similar to the arrangement in other species (Ramón y Cajal, 1960; Uga and Smelser, 1973; Robinson and Dreher, 1990; Dreher et al., 1992; Prada et al., 1998). The lateral territories of zebrafish MGs increased from the inner to outer retina: ILM, $28.6 \pm 3.6 \mu \mathrm{m}^{2}$; IPL, $62.6 \pm 6.4$ $\mu \mathrm{m}^{2}$; OPL, $89.6 \pm 9.1 \mu \mathrm{m}^{2}$; and OLM, $120.4 \pm 8.5 \mu \mathrm{m}^{2}$ (Fig. $1 \mathrm{~A}, C$, $\mathrm{SC})$.

In the gfap:GFP transgenic line (Bernardos and Raymond, 2006), all MGs are labeled by GFP expression (Fig. $1 B$; supplemental Movie 1, available at www.jneurosci.org as supplemental material). By photobleaching single MGs, the territory occupied only by the bleached cell was unmasked by loss of GFP signal, evident when images acquired before and after photobleaching were compared (Fig. $1 B$; supplemental Movie 2, available at www.jneurosci.org as supplemental material). In the OPL, IPL, and ILM, territories of individual MGs expressing GFP were similar to territories occupied by photobleached MGs, indicating that MG territories at these retinal depths are unlikely to overlap (Fig. 1C). However, in the OLM, the territories occupied by the processes of individual GFP-expressing MGs were significantly larger ( $p=0.024$ ) than those of photobleached MGs, suggesting that processes of neighboring MGs likely overlap by $\sim 20 \%$ in the OLM. Thus, like astrocytes (Bushong et al., 2002), in the synaptic layers of the retina, MGs occupy distinct spatial territories. This implies that individual synapses in the retina may be largely contacted by only a single glial cell.

\section{MG territories may be established by homotypic interactions}

To determine whether MG processes are intrinsically limited in their growth or establish their territories through MG-MG homotypic interactions, we cut the apical processes of a small number ( $4-8$ cells) of MGs at $4 \mathrm{dpf}$, one cell at a time using the multiphoton laser set to $750 \mathrm{~nm}$ (supplemental Movie 3, available at www.jneurosci.org as supplemental material). In some cases, cell bodies disappeared within $1 \mathrm{~h}$ after the process was cut, whereas in others, the cell body and apical process remained. For most ablations performed at these early stages, surrounding cells were not obviously perturbed in their morphology (supplemen- 
A

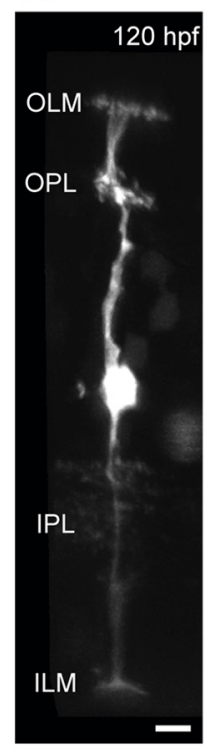

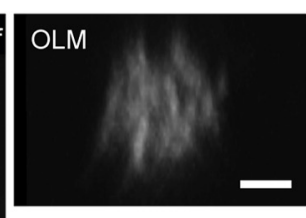
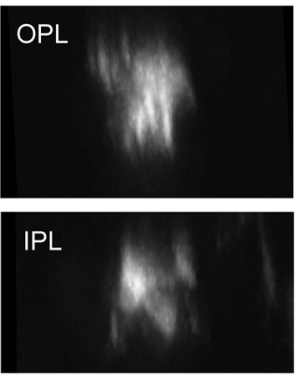

ILM

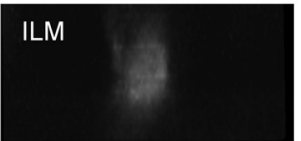

B

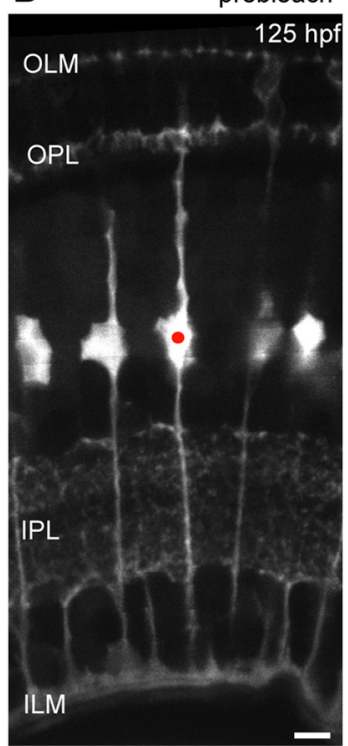

photobleached

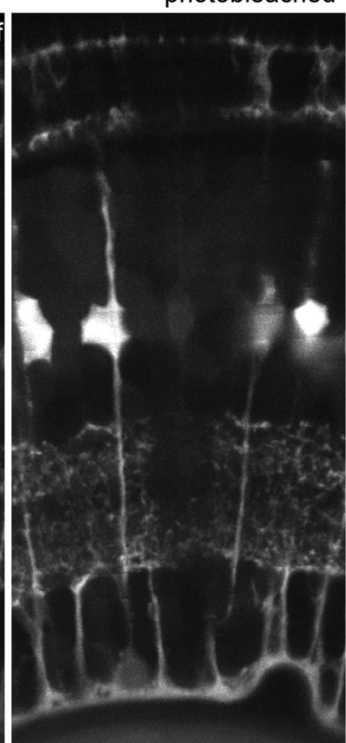

C
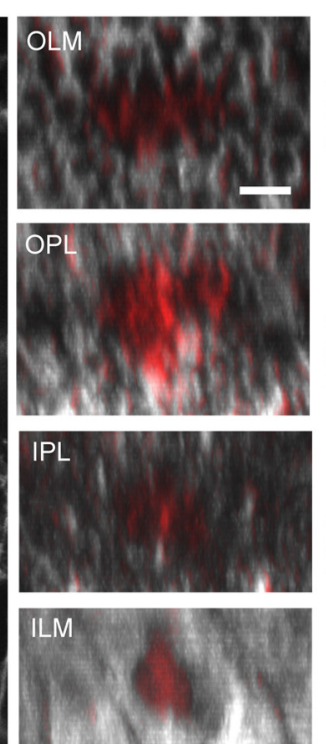
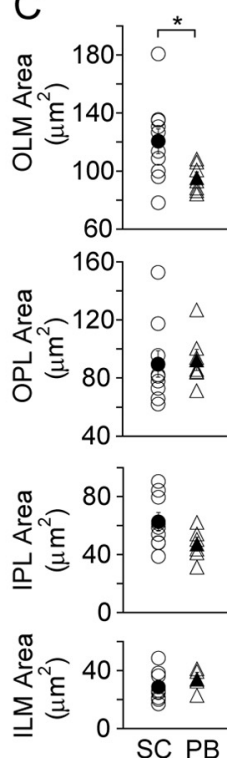

Figure 1. MG territories do not overlap in retinal synaptic layers. $A$, Maximum projection of a multiphoton image stack encompassing a single $M G$ at $120 \mathrm{hpf}$ labeled by transient expression of $G F P$. Orthogonal rotations (right column) of the image stack showing MG territories at the OLM and ILM and within the synaptic layers, the OPL and IPL. $\boldsymbol{B}$, A single MG (red dot) in the gfap:GFP transgenic line was photobleached using an IR laser. In orthogonal rotations of the MG territories at various retinal depths, the coverage of the photobleached cell is highlighted in red. The image stack before bleaching (pseudocolored red) was digitally overlaid with the image stack after photobleaching (grayscale) using AMIRA software. C, Territories occupied by a single MG obtained by transient GFP expression (SC) are compared to areas revealed upon photobleaching one cell (PB). ${ }^{*} p=0.024$. Open symbols indicate individual cells. Closed symbols indicate the mean. Error bars are SEM. Number of cells visualized by GFP and photobleaching respectively: $0 \mathrm{LM}(11,7), \operatorname{ILM}(9,4), 0 \mathrm{PL}(10,7), \operatorname{IPL}(9,6)$. All scale bars, $5 \mu \mathrm{m}$.

tal Fig. S1 A, available at www.jneurosci. org as supplemental material). However, in some instances, MG processes in the OPL and OLM merged and photoreceptor somata crossed the OLM (supplemental Fig. S1 $B$, available at www.jneurosci. org as supplemental material). Ablations leading to merged $M G$ territories in the OPL and OLM were not included for further analysis because retinal architecture was significantly altered in these instances. In ablations for which the OPL and OLM were maintained, time-lapse imaging revealed that the OPL was eventually completely covered by the processes of MGs surrounding the ablated region (supplemental Fig. S2, available at www. jneurosci.org as supplemental material). Targeted cells that retained their cell bodies did not regenerate an apical process. Furthermore, comparison of the number of MG somata in the field of view indicated that no new MGs were generated by cell division during the period of apparent $\mathrm{MG}$ process regrowth ( $n=35$ retinas), suggesting that MG processes extending into the ablated region arise from existing MGs.

If homotypic interactions regulate MG territory size, then MGs adjacent to the ablated cell should develop larger territories that account for the eventual coverage of the OPL days after ablation. Indeed, MGs bordering the ablation zone demonstrated enlarged, biased arbors atypical of normal MGs (Fig. 2A, compare with Fig. 1A), compared to cells in retinas of the same age without ablations
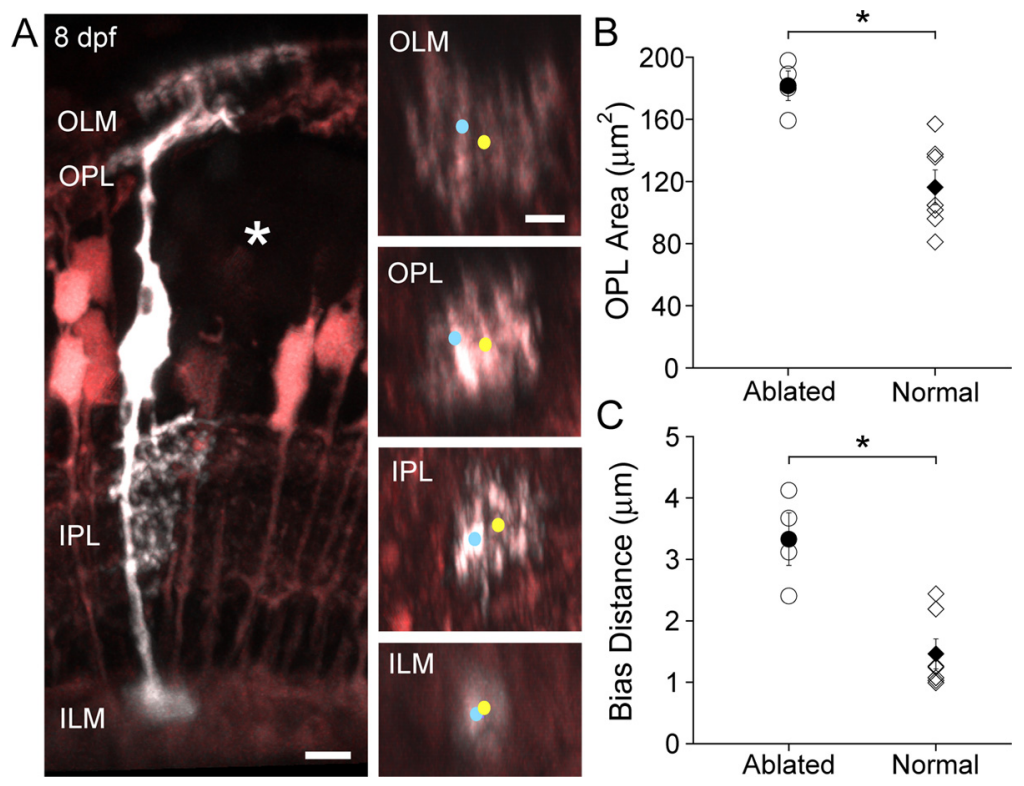

Figure 2. Homotypic interactions likely establish MG territories. A, An $8 \mathrm{dpf}$ MG expressing cerulean (grayscale) in the gfap:GFP transgenic background (pseudocolored pink) $5 \mathrm{~d}$ after an adjacent patch of $6 \mathrm{MG}$ s were ablated (asterisk). An enlarged arbor biased toward the ablated region is apparent. In orthogonal rotations, the apical and basal stalks are marked with a cyan dot and the centers of mass are marked with a yellow dot. Scale bars, $5 \mu \mathrm{m}$. B, Comparison of MG territory in the OPL between MGs bordering patches of ablated cells (such as the cell in $\boldsymbol{A}$ ) and normal MGs. ${ }^{*} p=0.0002$. C, Comparison of the distance from the apical stalk (blue dot) to the OPL territory center of mass (yellow dot) between MGs bordering ablation zone and normal MGs at $8 \mathrm{dpf} .{ }^{*} p=$ 0.02. B, C, Open symbols indicate individual cells. Closed symbols indicate the mean. Error bars are SEM.

( $p=0.006$ ) (Fig. 2B). Furthermore, MGs at the border of the ablation zone appear to extend processes toward the space previously occupied by the ablated cells. To quantify this apparent biased outgrowth across cells, we calculated the distance between the apical stalk (Fig. 2A, blue dot) and the center of mass of the OPL territory (Fig. $2 A$, yellow dot) for each cell bordering the 


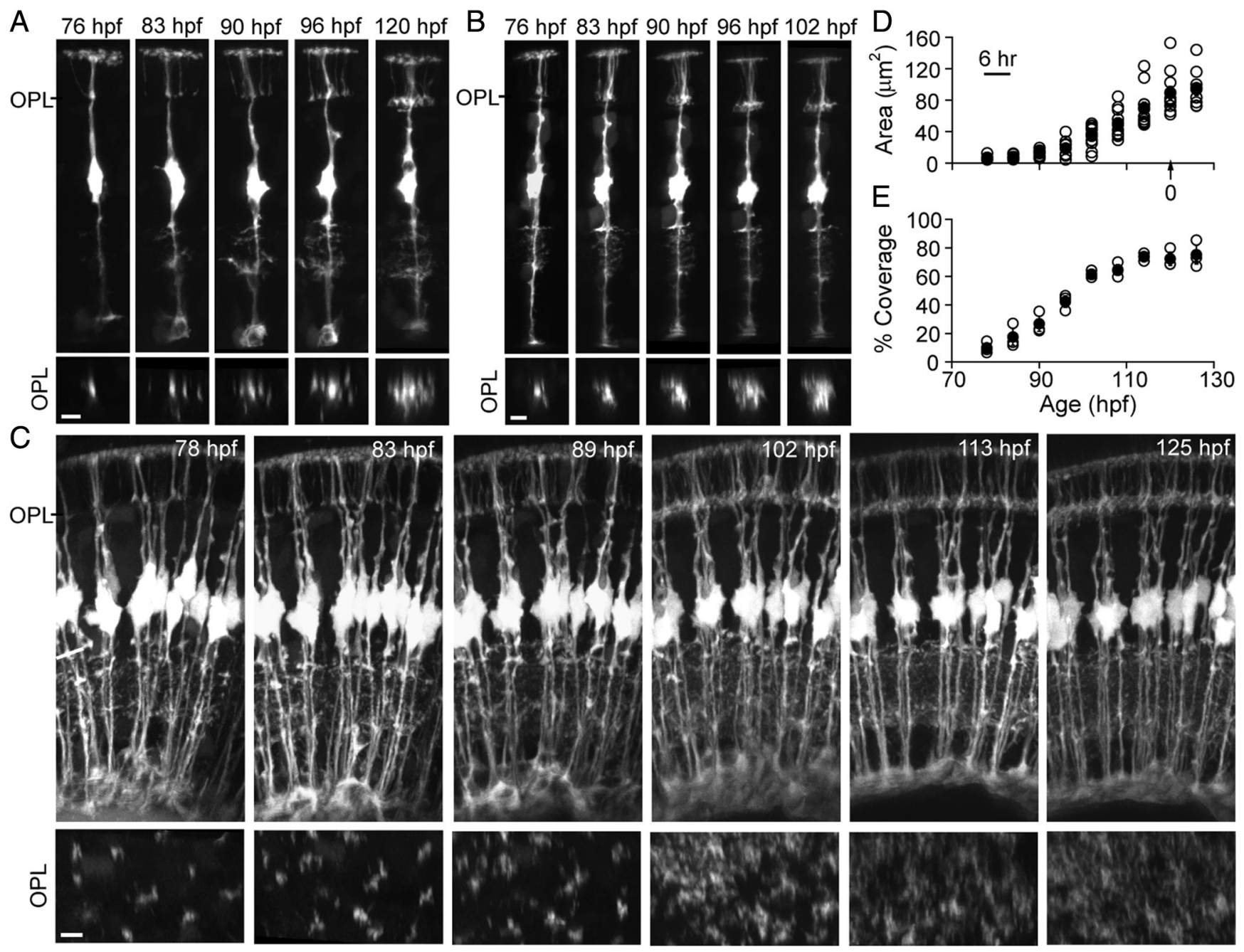

Figure 3. MG processes in the OPL are the last to mature. $A, B$, Time course of OPL territory development for two examples of MGs visualized by transient expression of GFP. Orthogonal rotations of each image stack at the level of the OPL (OPL image sequence) provide a lateral view of the MG territory at each time point. $C$, Time course of MG process infiltration into the OPL for the entire population of MGs imaged from the ventrotemporal retina. Orthogonal rotations show coverage of the OPL by elaborating MG processes. $D$, Time course of MG arbor elaboration within the OPL, normalized across multiple cells. Time "0" (arrow) is the age at which each cell first demonstrated a stable and maximal arbor. Maximal arbor formation occurred between 90 and $120 \mathrm{hpf}$. 0 pen symbols indicate individual cells. Closed symbols indicate the mean. Error bars are SEM. E, Time course of MG process occupancy within the OPL obtained by imaging MGs in the gfap:GFP transgenic line. MG process infiltration was calculated by counting the fraction of pixels above a manually set threshold. Open symbols indicate individual retinas. Closed symbols indicate the mean. Error bars are SEM. All scale bars, $5 \mu \mathrm{m}$.

ablation zone, and compared these distances with those obtained from MGs in nonablated retinas. The distance between the position of the stalk and the OPL arbor center of mass was significantly increased in cells bordering the ablation $(p=0.042)$ (Fig. $2 C)$. These data suggest that MG territories in the OPL are not inherently restricted in size, but are likely limited by interactions with adjacent MGs.

To ascertain whether the observed MG process extensions could have been due to reactive gliosis triggered by the laser ablations, we performed immunostaining for GFAP after ablation. Normally, zebrafish MGs express GFAP, and expression is concentrated in the basal half of the cell (supplemental Fig. S3B, available at www.jneurosci.org as supplemental material) (Bernardos and Raymond, 2006). We found that this pattern of endogenous expression was unchanged in MGs surrounding the ablation region, suggesting that massive gliosis was not triggered in the neighboring cells (supplemental Fig. S3, available at www. jneurosci.org as supplemental material). Thus, the filling in by MG processes as shown in Figure 2 is likely to be due to a relief of
MG-MG interactions that produce tiling of their territories in the synaptic layers.

\section{OPL process outgrowth is the last stage of MG} morphological maturation

Previous imaging studies have shown that a laminated plexus separating the developing photoreceptor layer from the INL is present by 45 hpf in zebrafish (Godinho et al., 2007). Our time-lapse imaging experiments showed that MG processes did not begin to elaborate in the OPL until after $76 \mathrm{hpf}$ (Fig. 3), $>1 \mathrm{~d}$ after the first signs of a laminated outer retinal plexus. Because in the gfap:GFP transgenic line MGs are labeled by cytosolic filling with GFP, it is possible that some fine processes of the MGs were not visualized. We thus colabeled GFP-expressing MGs with DiI, a lipophilic dye that labels cell membranes. Colabeling of DiI with GFP confirmed a lack of MG processes within the OPL at $77 \mathrm{hpf}$ (supplemental Fig. S4, available at www.jneurosci.org as supplemental material).

The relatively late filling in of the OPL by MG processes was clearly evident when process outgrowth of the complete popula- 
tion of MGs was followed (Fig. 3C). A stable MG arbor was established in the OPL $\sim 36 \mathrm{~h}$ from the initiation of outgrowth, and the final size of the arbor within the OPL varied between 60 and $140 \mu \mathrm{m}^{2}$ (Fig. $3 D)$. Interestingly, MG process extension within the OPL appeared synchronized among cells in the field of view. The time taken for individual arbors to reach a stable size matched the time period the population of MG arbors needed to occupy maximal coverage in the OPL (Fig. 3E). Interestingly, rudimentary lateral processes were already present in the IPL before processes appeared in the OPL (Fig. $3 A-C)$. When we examined GFP expression in gfap:GFP fish ( $n=3$ animals) between 55 and $70 \mathrm{hpf}$, it was evident that a few short processes protruded from the basal stalks of MGs at $55 \mathrm{hpf}$, which increased in their occupancy of the IPL over the subsequent day (supplemental Fig. S5, available at www.jneurosci.org as supplemental material) (Fig. 3).

\section{Photoreceptor synapses are present before MG process infiltration}

Previous ultrastructural observations suggest that contacts between photoreceptors and inner retinal neurons are present in the precocious ventronasal patch of the zebrafish retina as early as $62 \mathrm{hpf}$ (Schmitt and Dowling, 1999). Since we observed that MG processes elaborated at the border between photoreceptors and inner retinal neurons relatively late in development, we determined whether or not synapses were already established in the OPL before MG process outgrowth at this retinal location outside the ventronasal patch where we performed our imaging.

In more mature retina, the site of postsynaptic dendritic invaginations into cone pedicles is evident even by light microscopy (Fig. $4 A, B$ ). To examine the presence of photoreceptor synapses before MG process elaboration in the OPL, it was necessary to label cone photoreceptors early in development. We thus cloned promoter elements from $\operatorname{tr} \beta 2$ to drive expression of fluorescent proteins in cones. At $78 \mathrm{hpf}$, invaginations in fluorescent protein-expressing cone pedicles were also apparent due to the presence of a dark spot in the pedicle (Fig. 4C-E). To verify that these dark spots are sites of contact with postsynaptic cells, we labeled cone photoreceptor pedicles in transgenic zebrafish in which most ON bipolar cells (Schroeter et al., 2006) or most HCs (Parsons et al., 2009) are labeled by expression of MYFP (Fig. 4C,D). In all instances, cone photoreceptors apposed to bipolar cells (Fig. $4 C)(n=13)$ or HCs (Fig. $4 D)(n=52)$ possessed pedicles with invaginations of postsynaptic dendritic tips. Thus the dark spots within the cone pedicles represent invaginations at contact sites between the pho-

$\mathrm{E}$
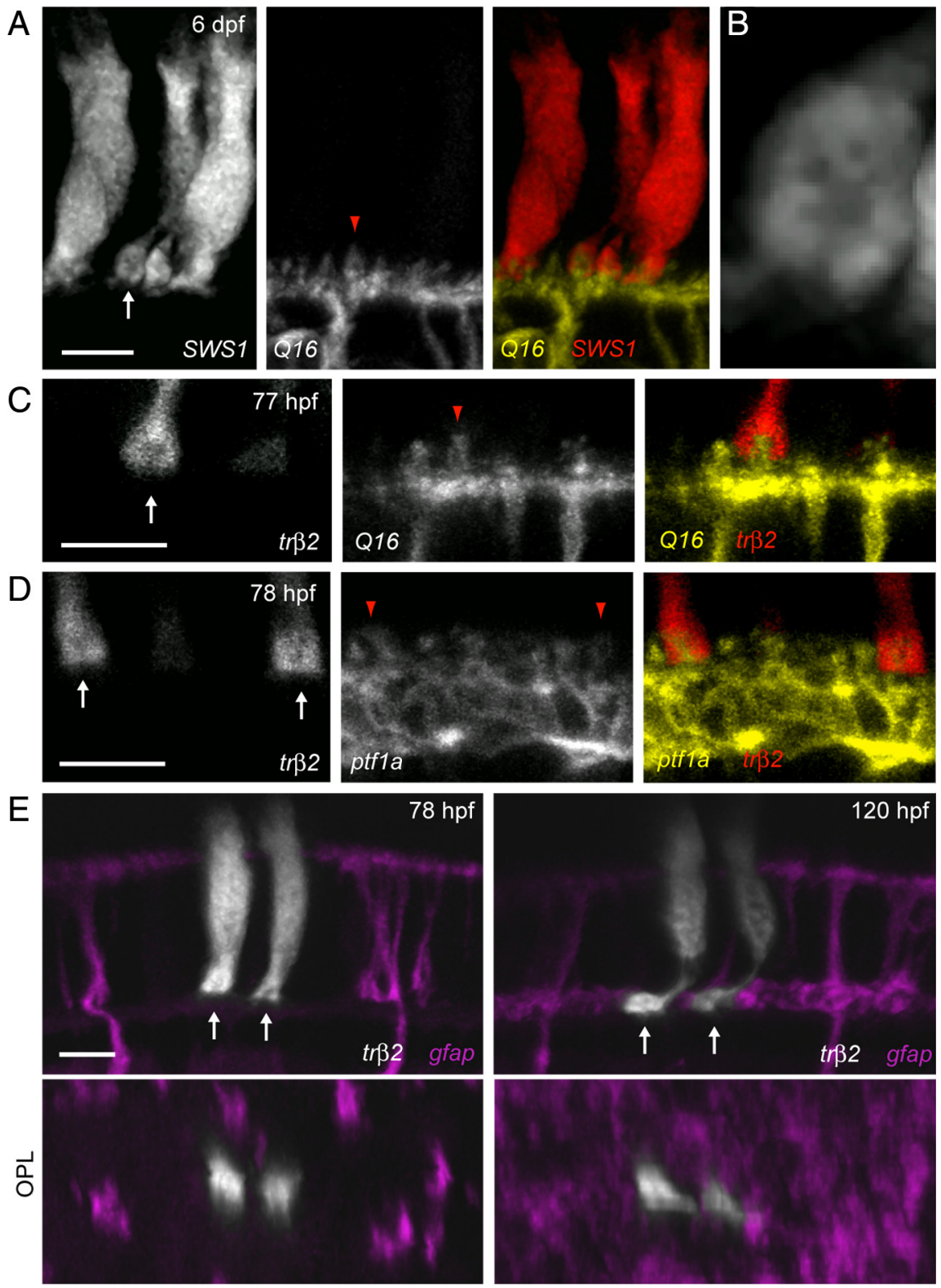

Figure 4. Cone pedicle invagination precedes MG process arrival in the OPL. $A$, Cone photoreceptors expressing tdTomato driven by the SWS1 promoter in the background of the Q16 line labeling ON bipolar cells. Arrow points to a cone pedicle. $\boldsymbol{B}$, High magnification of the cone terminal in $\boldsymbol{A}$ (arrow) reveals the presence of a dark region within the pedicle, suggestive of the location of the invaginations of postsynaptic $\mathrm{HC}$ and bipolar cell dendrites. C, Insertion of a bipolar cell (yellow, Q16) dendritic tip (red arrowhead) into a cone photoreceptor (red, $\operatorname{tr} \beta 2$ ) pedicle (arrow) at an age (77 hpf) where MG processes are absent from the OPL. $D$, Insertion of HC (yellow, ptf1a) dendritic tips (red arrowheads) into cone photoreceptor (red, tr $\beta 2$ ) pedicles (arrows) at an age (78 hpf) where MG processes have not infiltrated the OPL. E, A pair of cone photoreceptors (grayscale) transiently expressing tdTomato at $78 \mathrm{hpf}$ demonstrate invaginations (dark spots) in their pedicles (arrows) before the arrival of $M G$ processes (magenta) in the OPL. Orthogonal rotations (OPL) clearly show that cone pedicles were not contacted by MG processes at this stage. The same cones and MGs imaged at an age when MG processes surround the cone pedicles (right). Cones were labeled by injecting tr $\beta 2$ : tdTomato plasmid into the transgenic line gfap:GFP. Scale bars, $5 \mu \mathrm{m}$.

toreceptors and their postsynaptic cells. Time-lapse observations subsequently revealed that invaginations existed before the arrival of MG processes in the OPL and persisted throughout the time course of MG process elaboration (Fig. $4 E$ ).

We then further determined whether contact between photoreceptor pedicles and dendritic tips were synaptic in nature by immunostaining for presynaptic and postsynaptic elements in the OPL. Photoreceptor synaptic ribbons were labeled using antibodies directed against RIBEYEb (Obholzer et al., 2008) in 

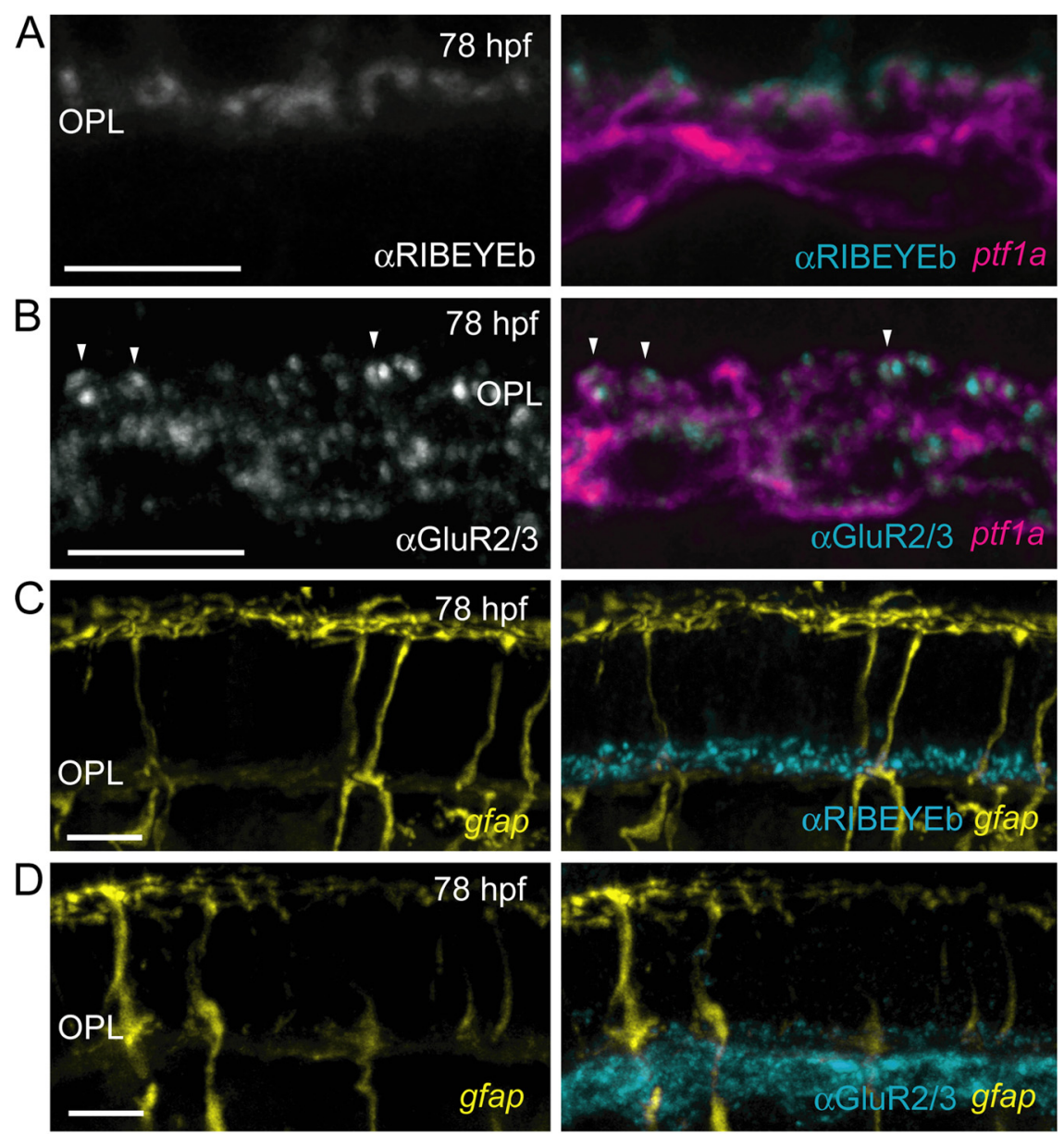

E
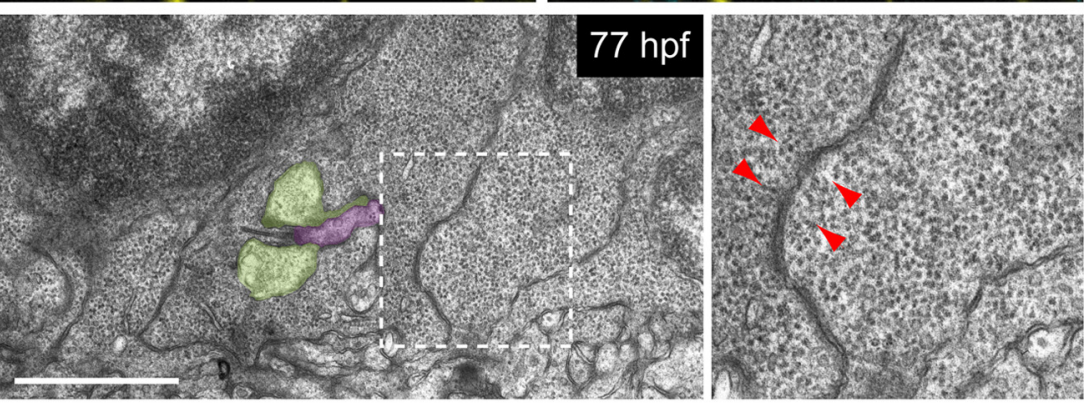

F
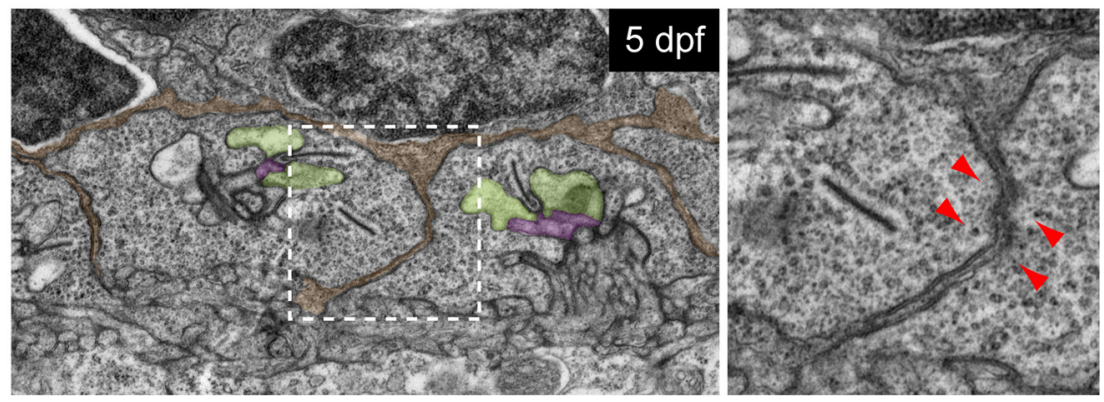

Figure 5. Synaptic components are present in the OPL before ingrowth of MG processes. HCs visualized in the ptf1a:G4VP16; UAS:MYFP double transgenic line and MGs in the gfap:GFP fish. Immunostaining was performed on $20 \mu \mathrm{m}$ frozen sections. $\boldsymbol{A}, \mathrm{HC}$ (magenta) dendritic tips are directly apposed to photoreceptor synaptic ribbons that are immunoreactive for RIBEYEb (grayscale, cyan). $\boldsymbol{B}, \mathrm{HC}$ (magenta) dendritic tips in the OPL contain puncta that are immunoreactive for GluR2/3 (grayscale, cyan, examples indicated by arrowheads). C, RIBEYEb immunoreactivity (cyan) in the OPL precedes the arrival of MG processes (yellow). A relatively dim, diffuse autofluorescent signal is apparent in the OPL of frozen sections in contrast to the bright lateral processes of MGs when they occupy the OPL (see Fig. 1). D, GluR2/3 immunoreactivity (cyan) in the OPL is present before MG process (yellow) arrival. $\boldsymbol{E}$, Electron micrographs of the OPL at $77 \mathrm{hpf}$ show the presence of ribbons associated with vesicles and triad invagination. Higher magnification of the region within the white box (right panel) demonstrates that photoreceptor pedicle plasma membranes are directly apposed to one another (red arrowheads). $\boldsymbol{F}$, Ultrastructure of the OPL at $5 \mathrm{dpf}$. Processes of presumptive MGs (pseudocolored transgenic fish where most HCs express MYFP. Nearly all HC dendritic tips examined were directly apposed to RIBEYEb immunofluorescent puncta in the OPL (Fig. 5A) (191 of 192 dendritic tips). An antibody directed against glutamate receptor subunits 2 and 3 (GluR2/3) was also used to identify postsynaptic terminals in HCs (Yazulla and Studholme, 2001). All HC dendritic tips examined contained GluR2/3-immunoreactive puncta (Fig. 5B) $(n=198)$. Thus, both presynaptic and postsynaptic proteins were localized to points of contact between photoreceptors and HCs. We then labeled presynaptic and postsynaptic elements at these early ages in transgenic fish where all MGs are labeled. Abundant punctate labeling of photoreceptor ribbons in the OPL was observed in regions lacking MG processes using the RIBEYEb antibody (Fig. 5C). GluR2/3 puncta were also apparent in regions of the OPL where MG processes were absent (Fig. 5D). Both RIBEYEb and GluR2/3 immunoreactivity was present as a dense "monolayer" in the OPL similar to patterns of synaptic protein expression in the adult zebrafish (Yazulla and Studholme, 2001). Thus, presynaptic and postsynaptic proteins are both present in the OPL and localized at $\mathrm{HC}$ dendritic tips before the elaboration of $M G$ processes.

To confirm that photoreceptor synapses are present in the OPL before MG process infiltration, we performed electron microscopy on age-matched embryos. The triad synapses of photoreceptors with ribbons apposed to horizontal cell and bipolar cell dendrites are evident at $5 \mathrm{dpf}$ (Fig. $5 F$; supplemental Fig. S6, available at www.jneurosci.org as supplemental material). In $77 \mathrm{hpf}$ fish retinas ( $n=2$ animals), photoreceptor pedicles containing ribbons with vesicles and examples of triad synapses were also observed (Fig. $5 E)$. At this early age, 24 of 32 cone terminals we followed through serial sections had one or more ribbons (see Materials and Methods and supplemental Fig. S6, available at www.jneurosci.org as supplemental material). This is likely to represent an undersampling of the total population of photoreceptors with rib-

$\leftarrow$

orange) ensheathe photoreceptor pedicles (red arrowheads). Higher magnification of the region within the white box (right panel) demonstrates that MG processes separate photoreceptor pedicle plasma membranes (red arrowheads). HC dendritic tips are shaded green, bipolar cell dendritic tips are shaded purple. Scale bars: $A-D, 5 \mu \mathrm{m} ; \boldsymbol{E}, \boldsymbol{F}, 1 \mu \mathrm{m}$. 
bons at $77 \mathrm{hpf}$, since it was not readily possible to completely reconstruct each photoreceptor within the field.

We also noted that at 76-77 hpf, cone pedicles abutted one another without much, if any, ensheathing MG processes (Fig. 5E, arrowheads). In contrast, at 5 $\mathrm{dpf}$, photoreceptor pedicles were consistently separated and ensheathed by processes, presumably of MGs (Fig. 5F, pseudocolored orange). Together, our light and electron microscopy observations demonstrate that photoreceptor synapses are present before MG processes arrive in the OPL.

\section{MG contact is not critical for stabilizing early synapses in the OPL}

Although we demonstrated the presence of synapses in the OPL before the arrival of MG processes, some of these contacts may be nascent and unstable, raising the possibility that MG contact is important for the stabilization of the early synaptic triads. One way to track the stability of "contact" between cones and their postsynaptic targets is to monitor the behavior of the dendritic tips of HCs or those of bipolar cells over time. We focused on the dynamic behavior of $\mathrm{HC}$ dendritic tips that are somewhat larger than those of bipolar cells. Because the dendritic tips of HCs at $78 \mathrm{hpf}$ expressed GluR2/3 and were apposed to the presynaptic ribbon marker RIBEYEb, it was likely that dendritic tips of the HCs were postsynaptic in nature. Time-lapse imaging of individual HCs showed that their dendritic tips underwent remodeling over $2 \mathrm{~d}$, during the period of MG elaboration in the OPL (Fig. 6A). Both persistent tips (Fig. $6 \mathrm{~A}$, arrowheads) and newly formed tips (Fig. 6A, empty arrows) were observed. Tips that persisted for at least $18 \mathrm{~h}$ and were present at the final time point were defined as "stable" (Fig. 6A, green arrowheads), whereas tips that retracted during the $48 \mathrm{~h}$ time-lapse recording were defined as "transient" (Fig. 6A, red arrowhead).

During these stages of $\mathrm{HC}$ dendritic tip remodeling, contact between HC dendritic tips and MG processes could be clearly observed (supplemental Movie 4, available at www.jneurosci.org as supplemental material). To determine whether $\mathrm{HC}$ dendritic tip stability was influenced by contact with MG processes, we compared the duration of transient $\mathrm{HC}$ dendritic tips to the time course of MG contact onto stable tips. If contact with MGs stabilizes HC dendritic tips, contact with MG processes must occur before tip retraction. The majority of transient tips retracted after only a single time point (Fig. $6 \mathrm{~B}$ ), and possessed an average lifetime of $9.5 \pm 0.7 \mathrm{~h}(n=65)$. Although most stable HC dendritic tips were contacted by MG processes ( 41 of 53 ), the average stable tip received contact from $M G$ processes only some $17.4 \pm 1.6 \mathrm{~h}$ after its formation (Fig. $6 C)(n=41)$. Furthermore, 12 stable tips did not receive contact from MGs during the course of these experiments (total duration $26.5 \pm 1.2 \mathrm{~h}$ ), suggesting an even later time course for MG-HC contact formation. Thus, on aver- age, stable tips were contacted by MG processes much later after their formation than the average time for which transient tips persisted. These results indicate that MG contact is unlikely to be necessary for the stabilization of newly formed HC dendritic tips.

To directly demonstrate that components of the developing photoreceptor-HC circuit can form and be maintained in the absence of MG processes, we performed MG laser ablations at 4 dpf before process elaboration in the OPL. We observed HC process stability as well as maintenance of cone photoreceptor pedicle invaginations in regions lacking $\mathrm{MG}$ processes. Areas lacking MGs persisted for 1-5 d after ablations (supplemental Fig. S2, available at www.jneurosci.org as supplemental material).

MG ablations were performed in transgenic zebrafish in which all HCs and MGs were labeled to observe the local effects of MG removal on HC dendritic tip stability. Following MG ablations, $\mathrm{HC}$ dendritic tips were clearly visible even in large areas lacking MG processes (Fig. 7A, arrowheads). HC dendritic tip densities in regions lacking $M G$ processes were unchanged relative to adjacent regions where MG processes were maintained (Fig. $7 B)(p=0.72)$. Therefore, removal of MG processes from the OPL had no apparent effect on the maintenance of HC dendritic tips for at least up to $3 \mathrm{~d}$ after ablation.

We then examined the effects of MG process removal from the OPL on the stability of cone contacts by observing pedicle invagi- 

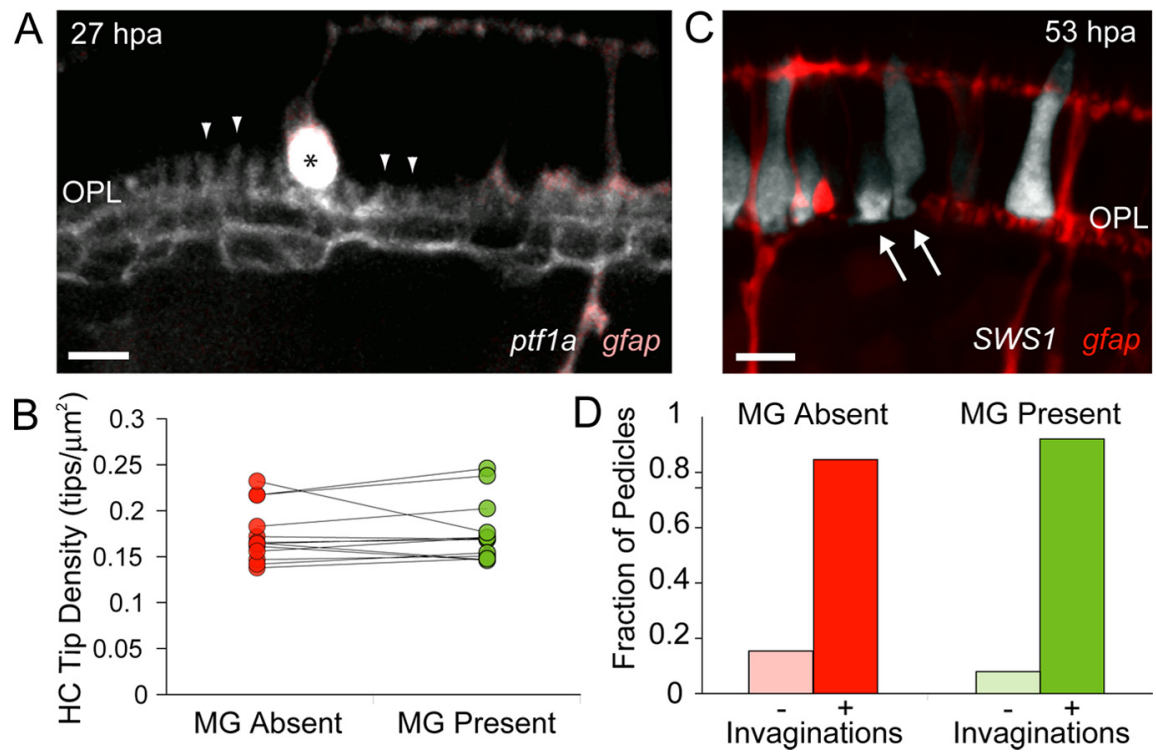

Figure 7. MG processes are not required for $\mathrm{HC}$ dendritic tip or cone photoreceptor pedicle stability. $\boldsymbol{A}$, Six MGs were ablated at $4 \mathrm{dpf}$ in a triple transgenic line labeling HCs (grayscale, ptf1a:G4VP16; UAS:MYFP) and MGs (pink, gfap:GFP). Examples of HC dendritic tips remaining in areas that lack MG processes are indicated by arrowheads. Asterisk indicates swollen debris of apical $M G$ process. $\boldsymbol{B}, \mathrm{HC}$ dendritic tip densities were compared within the field of view for regions where MGs were absent (red dots) and regions where they were present (green dots). Lines connect measurements from the same field (an ablation experiment). Analysis was performed only in regions large enough to contain at least five dendritic tips, and were pooled across all time points after ablation (1-4 d after ablation). $n=13$ ablated regions. C, Six MGs were ablated at $4 \mathrm{dpf}$ in retinas where UV cones transiently expressed tdTomato (grayscale) in the gfap:GFP transgenic line (red). UV-cone photoreceptor invaginations (dark spot within the pedicles) are indicated with arrows. $\boldsymbol{D}$, Fraction of UV-cone photoreceptor pedicles that collapsed $(-)$ or remained invaginated $(+)$ in areas lacking MG processes (red, $n=26$ ) or where MG processes were present (green, $n=38$ ). Scale bars, $5 \mu \mathrm{m}$.

nations following MG ablation. UV cone photoreceptors transiently expressing tdTomato were examined in the gfap:GFP transgenic line where patches of six MGs were ablated. In regions lacking MG processes, photoreceptors expressing tdTomato still contained synaptic invaginations in their pedicles (Fig. 7C). There was no significant difference in the fraction of photoreceptor pedicles containing or lacking invaginations between regions in the OPL with or without MG processes (Fig. $7 D)(p=0.43)$. Together, these results strongly indicate that MG structural contributions to the OPL and contacts with neuronal synaptic elements are not required for the development and early maintenance of OPL circuits.

\section{Discussion}

Using an in vivo imaging approach, we found that MGs occupy exclusive territories in the synaptic layers of the retina. MG territories are likely established via homotypic interactions and develop asynchronously, with processes in the OPL being the last to elaborate. MG processes in the OPL arrive after the formation of photoreceptor and $\mathrm{HC}$ synaptic structures but during the period when $\mathrm{HC}$ dendritic tips are still motile. $\mathrm{HC}$ dendritic tip stability is not influenced by contact with MGs, and removal of MGs from small patches of the developing retina had no effect on the maintenance of HC dendritic tip density or photoreceptor pedicle stability. Therefore, we conclude that glial contact does not play a critical role in the formation and early stability of outer retinal circuits.

\section{Factors influencing the development of MG territories}

Homotypic interactions are important for the formation of tiled mosaics in developing peripheral sensory neurons of zebrafish (Sagasti et al., 2005), leech (Gan and Macagno, 1995), and Dro- sophila (Grueber et al., 2003; Sugimura et al., 2003). Migrating HCs in the mouse retina display a transient stage with exclusive territories that are thought to underlie proper positioning of somata (Huckfeldt et al., 2009). Brain astrocytes also form similar non-overlapping territories (Bushong et al., 2002), but the mechanisms by which these territories develop are largely unknown. Processes of neighboring protoplasmic astrocytes transiently overlap during development (Bushong et al., 2004), lending support to the idea that contact-mediated homotypic interactions are important for setting up exclusive glial domains. More rapid timelapse imaging of zebrafish oligodendrocyte precursor cells in vivo revealed that when the processes of these glia come into contact, they retract within minutes (Kirby et al., 2006), suggesting that homotypic contacts mediate process removal.

We demonstrated here that, like protoplasmic astrocytes, MG territories are exclusive or nearly exclusive throughout the retinal synaptic layers, in contrast to previous experiments in the rabbit that observed overlapping processes of neighboring HRP-labeled MGs (Robinson and Dreher, 1990). This discrepancy may have been due to more accurate labeling methods, or possible species differences, as tiling of teleost MGs has not been investigated previously. Our observation of MG OPL territories expanding toward regions where neighboring MGs had been removed further supports the claim that homotypic interactions, and not cell intrinsic programs, are important for establishing glial territories. Such a mechanism for elaboration, where processes fill nearby space until meeting their neighbors, would be an efficient way to establish full coverage across the retina where MG cell density varies along a centro-peripheral gradient (Dreher et al., 1992), while maintaining exclusive territories of individual cells.

Our in vivo imaging study also revealed that MGs form each of their four distinct territories in different retinal layers independently. Previous experiments labeling MGs with Golgi impregnation methods have observed that MG territories mature along a basal to apical sequence (Ramón y Cajal, 1960; Morest, 1970). Conversely, we found that the OLM appeared to be the first territory to fully differentiate (Fig. 5A,B). Relatively early in development, contacts between the apical processes of MGs and photoreceptor inner segments ( $50 \mathrm{hpf}$ ) have been observed in the zebrafish retina by electron microscopy (Schmitt and Dowling, 1999), supporting our findings. The late differentiation of MG processes in the OPL, after synaptic elements have formed, would imply that MG processes either target existing synapses in the OPL or passively grow throughout the synaptic layer and form associations with synapses. The outgrowth of $\mathrm{MG}$ processes in the OPL was highly directed to the neuropil (Fig. 3), raising the possibility of a targeting factor. Indeed, slice cultures of mature brainstem showed that regions of rapid astrocytic filopodial remodeling occur exclusively around active synapses labeled by FM dyes (Hirrlinger et al., 2004), demonstrating that astroglia can differentiate between synaptic and nonsynaptic zones. As such, 
the onset of synaptic activity in the OPL may guide MG process outgrowth.

\section{MG contact and its role in the development of outer retinal circuits}

There is abundant evidence in support of astroglial contact playing an important role in promoting synapse formation. In dissociated cocultures of neurons and astrocytes, significant increases in the number of presynaptic sites and the amplitude and number of EPSCs specifically in regions where postsynaptic cells are contacted by astrocytes were observed (Hama et al., 2004). Furthermore, in hippocampal slice cultures, astrocytic filopodia can stabilize developing dendritic spines through ephrin signaling (Nishida and Okabe, 2007). Blocking astrocytic process motility with dominant-negative Rac led to a decrease in the number of spines; however, dendritic filopodia that grew over longer distances than normal to contact astrocytes were just as likely to develop into spines as in normal controls (Nishida and Okabe, 2007). This synapse promoting activity is not limited to excitatory connections or projection neurons. The intensity of GABA signaling is significantly upregulated by astrocytic contact in dissociated hippocampal cultures (Liu et al., 1996).

In contrast to these and other examples (Reddy et al., 2003), our experiments showed that during normal development, synapses are present in the outer retinal synaptic layer before the arrival of MG processes at $\sim 76 \mathrm{hpf}$. These synapses are likely to be functional because the first visually evoked responses in zebrafish occur between 68 and 72 hpf (Branchek and Bremiller, 1984; Easter and Nicola, 1996), and optokinetic responses (which also require maturation of the extraocular muscles) are detected as early as $73 \mathrm{hpf}$ (Easter and Nicola, 1996). In addition, the removal of MGs had no effects on the stability of presynaptic cone photoreceptor pedicles or postsynaptic $\mathrm{HC}$ dendritic tips. In the zebrafish inner retina, however, MGs have rudimentary processes in the IPL at $55 \mathrm{hpf}$ (supplemental Fig. S5, available at www. jneurosci.org as supplemental material), $\sim 5 \mathrm{~h}$ before the first conventional synapses are observed by EM (Schmitt and Dowling, 1999). Thus, it remains possible that unlike the OPL, IPL synaptogenesis could be influenced by glial contact.

Experiments examining neurons from a number of circuits have shown that diffusible factors from glia can positively regulate synaptogenesis, including cholesterol (Mauch et al., 2001), thrombospondins (Christopherson et al., 2005), and activitydependent neurotrophic factor (Blondel et al., 2000) from astrocytes, as well as tumor necrosis factor $\alpha$ (Beattie et al., 2002) and transforming growth factor $\beta$ (Feng and Ko, 2008) from Schwann cells. Astrocyte-conditioned medium has also been shown to increase the number of interneuronal inhibitory synapses on cultured rat hippocampal neurons by indirectly upregulating BDNF/TrkB signaling from local neurons through an unidentified factor (Elmariah et al., 2005). Thus, although we found that direct structural contact between MGs and neuronal elements was not required for OPL synapse formation, MGs may still provide support for synapse development via diffusible factors. However, persistence of synaptic arrangements in the OPL despite local ablation of MGs suggest that OPL synapses are maintained for at least several days in the absence of local glia.

Even though contact with MGs is not important for initial synapse differentiation in the OPL, MGs may still be involved in reducing structural plasticity in developed circuits. Contrary to their role in promoting synaptogenesis during early development, astroglia are critical for limiting plasticity and removing exuberant synapses at later stages of development. For example, transplantation of immature astrocytes into adult cat visual cortex can reinstate plasticity in ocular dominance columns (Müller and Best, 1989) likely through digestion of chondroitin sulfate proteoglycans (Pizzorusso et al., 2002). In the cerebellum, limiting contact between Bergmann glia, the resident astroglia, and Purkinje cells leads to a maintenance of immature wiring patterns. Climbing fibers remain multiply innervated onto Purkinje neurons (Grosche et al., 1999; Iino et al., 2001), and the density of AMPA receptors remains high in the molecular layer (Lippman et al., 2008).

Given that MGs may thus be important for the long-term stability or refinement of neuronal circuitry, we attempted to use focal ablations to investigate the functions of MGs in more mature OPL circuits. However, this proved difficult to achieve with laser ablations. Although MGs could be killed at later ages, we found that their apical processes remained intact with normal morphology spanning the OPL and OLM for 1-5 d after ablation (data not shown). While these processes were gradually removed from the OPL, neighboring MG arbors elaborated to take over the unoccupied area. Thus, with laser ablations it was impossible to remove MG processes from the OPL or OLM once they had elaborated.

Finally, the overall stability of outer retinal circuits at the ages we examined is currently unknown. Although photoreceptor pedicles appeared stable within the developmental time frame we observed, some HCs were still adding new dendritic tips at the end of the recording period ( $5 \mathrm{dpf}$ ), albeit much less frequently than at the beginning of the experiments. It should also be noted that the cone photoreceptor mosaic is not yet regular over the ages we examined; thus, rearrangements in the positioning of cone photoreceptors must occur over the course of further development to create the highly regular array of cone photoreceptors present in the adult zebrafish (Raymond et al., 1995). Furthermore, rod photoreceptors are produced until $7 \mathrm{dpf}$ in the zebrafish retina (Fadool, 2003). This ongoing rearrangement of cone photoreceptors and addition of rod photoreceptors indicate that some OPL circuit remodeling is required for attaining the adult circuits. It is therefore likely that MGs at these ages do not restrict synaptic rearrangement. Our current study, however, suggests that the initial differentiation of photoreceptor circuits in the OPL is not dependent on direct structural associations with MGs.

\section{References}

Beattie EC, Stellwagen D, Morishita W, Bresnahan JC, Ha BK, Von Zastrow M, Beattie MS, Malenka RC (2002) Control of synaptic strength by glial TNFalpha. Science 295:2282-2285.

Bernardos RL, Raymond PA (2006) GFAP transgenic zebrafish. Gene Expr Patterns 6:1007-1013.

Blondel O, Collin C, McCarran WJ, Zhu S, Zamostiano R, Gozes I, Brenneman DE, McKay RD (2000) A glia-derived signal regulating neuronal differentiation. J Neurosci 20:8012-8020.

Brancheck T (1984) The development of photoreceptors in zebrafish. II. Function. J Comp Neurol 224:116-122.

Bushong EA, Martone ME, Jones YZ, Ellisman MH (2002) Protoplasmic astrocytes in CA1 stratum radiatum occupy separate anatomical domains. J Neurosci 22:183-192.

Bushong EA, Martone ME, Ellisman MH (2004) Maturation of astrocyte morphology and the establishment of astrocyte domains during postnatal hippocampal development. Int J Dev Neurosci 22:73-86.

Christopherson KS, Ullian EM, Stokes CC, Mullowney CE, Hell JW, Agah A, Lawler J, Mosher DF, Bornstein P, Barres BA (2005) Thrombospondins are astrocyte-secreted proteins that promote CNS synaptogenesis. Cell 120:421-433.

Dreher Z, Robinson SR, Distler C (1992) Muller cells in vascular and avascular retinae: a survey of seven mammals. J Comp Neurol 323:59-80. 
Easter SS Jr, Nicola GN (1996) The development of vision in the zebrafish, Danio rerio. Dev Biol 180:646-663.

Elmariah SB, Oh EJ, Hughes EG, Balice-Gordon RJ (2005) Astrocytes regulate inhibitory synapse formation via Trk-mediated modulation of postsynaptic GABA A receptors. J Neurosci 25:3638-3650.

Fadool JM (2003) Development of a rod photoreceptor mosaic revealed in transgenic zebrafish. Dev Biol 258:277-290.

Feng Z, Ko CP (2008) Schwann cells promote synaptogenesis at the neuromuscular junction via transforming growth factor- $\beta 1$. J Neurosci 28 : 9599-9609.

Friauf E, McConnell SK, Shatz CJ (1990) Functional synaptic circuits in the subplate during fetal and early postnatal development of cat visual cortex. J Neurosci 10:2601-2613.

Gan WB, Macagno ER (1995) Interactions between segmental homologs and between isoneuronal branches guide the formation of sensory terminal fields. J Neurosci 15:3243-3253.

Godinho L, Mumm JS, Williams PR, Schroeter EH, Koerber A, Park SW, Leach SD, Wong RO (2005) Targeting of amacrine cell neurites to appropriate synaptic laminae in the developing zebrafish retina. Development 132:5069-5079.

Godinho L, Williams PR, Claassen Y, Provost E, Leach SD, Kamermans M, Wong RO (2007) Nonapical symmetric divisions underlie horizontal cell layer formation in the developing retina in vivo. Neuron 56:597-603.

Grosche J, Matyash V, Möller T, Verkhratsky A, Reichenbach A, Kettenmann H (1999) Microdomains for neuron-glia interaction: parallel fiber signaling to Bergmann glial cells. Nat Neurosci 2:139-143.

Grueber WB, Ye B, Moore AW, Jan LY, Jan YN (2003) Dendrites of distinct classes of Drosophila sensory neurons show different capacities for homotypic repulsion. Curr Biol 13:618-626.

Haber M, Zhou L, Murai KK (2006) Cooperative astrocyte and dendritic spine dynamics at hippocampal excitatory synapses. J Neurosci 26: $8881-8891$

Hama H, Hara C, Yamaguchi K, Miyawaki A (2004) PKC signaling mediates global enhancement of excitatory synaptogenesis in neurons triggered by local contact with astrocytes. Neuron 41:405-415.

Hirrlinger J, Hülsmann S, Kirchhoff F (2004) Astroglial processes show spontaneous motility at active synaptic terminals in situ. Eur J Neurosci 20:2235-2239.

Huang CJ, Tu CT, Hsiao CD, Hsieh FJ, Tsai HJ (2003) Germ-line transmission of a myocardium-specific GFP transgene reveals critical regulatory elements in the cardiac myosin light chain 2 promoter of zebrafish. Dev Dyn 228:30-40.

Huckfeldt RM, Schubert T, Morgan JL, Godinho L, Di Cristo G, Huang ZJ, Wong RO (2009) Transient neurites of retinal horizontal cells exhibit columnar tiling via homotypic interactions. Nat Neurosci 12:35-43.

Iino M, Goto K, Kakegawa W, Okado H, Sudo M, Ishiuchi S, Miwa A, Takayasu Y, Saito I, Tsuzuki K, Ozawa S (2001) Glia-synapse interaction through $\mathrm{Ca} 2+$-permeable AMPA receptors in Bergmann glia. Science 292:926-929.

Kawakami K (2004) Transgenesis and gene trap methods in zebrafish by using the Tol2 transposable element. Methods Cell Biol 77:201-222.

Kawakami K, Shima A (1999) Identification of the Tol2 transposase of the medaka fish Oryzias latipes that catalyzes excision of a nonautonomous Tol2 element in zebrafish Danio rerio. Gene 240:239-244.

Kirby BB, Takada N, Latimer AJ, Shin J, Carney TJ, Kelsh RN, Appel B (2006) In vivo time-lapse imaging shows dynamic oligodendrocyte progenitor behavior during zebrafish development. Nat Neurosci 9:1506-1511.

Köster RW, Fraser SE (2001) Tracing transgene expression in living zebrafish embryos. Dev Biol 233:329-346.

Kwan KM, Fujimoto E, Grabher C, Mangum BD, Hardy ME, Campbell DS, Parant JM, Yost HJ, Kanki JP, Chien CB (2007) The Tol2kit: a multisite gateway-based construction kit for Tol2 transposon transgenesis constructs. Dev Dyn 236:3088-3099.

Lippman JJ, Lordkipanidze T, Buell ME, Yoon SO, Dunaevsky A (2008) Morphogenesis and regulation of Bergmann glial processes during Purkinje cell dendritic spine ensheathment and synaptogenesis. Glia 56:1463-1477.

Liu QY, Schaffner AE, Li YX, Dunlap V, Barker JL (1996) Upregulation of $\mathrm{GABA}_{\mathrm{A}}$ current by astrocytes in cultured embryonic rat hippocampal neurons. J Neurosci 16:2912-2923.

Livet J, Weissman TA, Kang H, Draft RW, Lu J, Bennis RA, Sanes JR, Licht- man JW (2007) Transgenic strategies for combinatorial expression of fluorescent proteins in the nervous system. Nature 450:56-62.

Mauch DH, Nägler K, Schumacher S, Göritz C, Müller EC, Otto A, Pfrieger FW (2001) CNS synaptogenesis promoted by glia-derived cholesterol. Science 294:1354-1357.

Morest DK (1970) The pattern of neurogenesis in the retina of the rat. Z Anat Entwicklungsgesch 131:45-67.

Müller CM (1990) Dark-rearing retards the maturation of astrocytes in restricted layers of cat visual cortex. Glia 3:487-494.

Müller CM, Best J (1989) Ocular dominance plasticity in adult cat visual cortex after transplantation of cultured astrocytes. Nature 342:427-430.

Nishida H, Okabe S (2007) Direct astrocytic contacts regulate local maturation of dendritic spines. J Neurosci 27:331-340.

Obholzer N, Wolfson S, Trapani JG, Mo W, Nechiporuk A, Busch-Nentwich E, Seiler C, Sidi S, Söllner C, Duncan RN, Boehland A, Nicolson T (2008) Vesicular glutamate transporter 3 is required for synaptic transmission in zebrafish hair cells. J Neurosci 28:2110-2118.

Parsons MJ, Pisharath H, Yusuff S, Moore JC, Siekmann AF, Lawson N, Leach SD (2009) Notch-responsive cells initiate the secondary transition in larval zebrafish pancreas. Mech Dev 126:898-912.

Pizzorusso T, Medini P, Berardi N, Chierzi S, Fawcett JW, Maffei L (2002) Reactivation of ocular dominance plasticity in the adult visual cortex. Science 298:1248-1251.

Prada FA, Quesada A, Dorado ME, Chmielewski C, Prada C (1998) Glutamine synthetase (GS) activity and spatial and temporal patterns of GS expression in the developing chick retina: relationship with synaptogenesis in the outer plexiform layer. Glia 22:221-236.

Ramón y Cajal S (1960) Studies on vertebrate neurogenesis. Reprint (Guth L, translator). Springfield, IL: Thomas.

Ransom B, Behar T, Nedergaard M (2003) New roles for astrocytes (stars at last). Trends Neurosci 26:520-522.

Raymond PA, Barthel LK, Curran GA (1995) Developmental patterning of rod and cone photoreceptors in embryonic zebrafish. J Comp Neurol 359:537-550.

Reddy LV, Koirala S, Sugiura Y, Herrera AA, Ko CP (2003) Glial cells maintain synaptic structure and function and promote development of the neuromuscular junction in vivo. Neuron 40:563-580.

Ren JQ, McCarthy WR, Zhang H, Adolph AR, Li L (2002) Behavioral visual responses of wild-type and hypopigmented zebrafish. Vision Res 42:293-299.

Robinson SR, Dreher Z (1990) Muller cells in adult rabbit retinae: morphology, distribution and implications for function and development. J Comp Neurol 292:178-192.

Rochefort N, Quenech'du N, Ezan P, Giaume C, Milleret C (2005) Postnatal development of GFAP, connexin43 and connexin30 in cat visual cortex. Brain Res Dev Brain Res 160:252-264.

Sagasti A, Guido MR, Raible DW, Schier AF (2005) Repulsive interactions shape the morphologies and functional arrangement of zebrafish peripheral sensory arbors. Curr Biol 15:804-814.

Schmitt EA, Dowling JE (1999) Early retinal development in the zebrafish, Danio rerio: light and electron microscopic analyses. J Comp Neurol 404:515-536.

Schroeter EH, Wong RO, Gregg RG (2006) In vivo development of retinal ON-bipolar cell axonal terminals visualized in nyx::MYFP transgenic zebrafish. Vis Neurosci 23:833-843.

Stevens B (2008) Neuron-astrocyte signaling in the development and plasticity of neural circuits. Neurosignals 16:278-288.

Sugimura K, Yamamoto M, Niwa R, Satoh D, Goto S, Taniguchi M, Hayashi S, Uemura T (2003) Distinct developmental modes and lesion-induced reactions of dendrites of two classes of Drosophila sensory neurons. J Neurosci 23:3752-3760.

Takechi M, Hamaoka T, Kawamura S (2003) Fluorescence visualization of ultraviolet-sensitive cone photoreceptor development in living zebrafish. FEBS Lett 553:90-94.

Uga S, Smelser GK (1973) Electron microscopic study of the development of retinal Mullerian cells. Invest Ophthalmol 12:295-307.

Ullian EM, Sapperstein SK, Christopherson KS, Barres BA (2001) Control of synapse number by glia. Science 291:657-661.

Ullian EM, Harris BT, Wu A, Chan JR, Barres BA (2004) Schwann cells and astrocytes induce synapse formation by spinal motor neurons in culture. Mol Cell Neurosci 25:241-251.

Yazulla S, Studholme KM (2001) Neurochemical anatomy of the zebrafish retina as determined by immunocytochemistry. J Neurocytol 30:551-592. 